\title{
Characteristic Parameter Estimation of Internal Waves Based on Pitch Arc Fitting and HHT
}

\author{
Jie Chen \\ Navy Submarine Acadamy \\ Biao Chen \\ Navy Submarine Acadamy
}

\author{
Jifeng Yang \\ Navy Submarine Acadamy \\ Suqin $\mathrm{Xu}$ \\ Submarine Acadamy
}

\begin{abstract}
In this paper the wave direction and the wave length of the internal wave will be extracted with a novel method, which will combine the merit of artificial allocation and the Hilber Huang Transform. The Internal wave in SAR image is firstly drew with three points along the front of the internal wave, then the arithmetic proposed in this paper will automatically calculate the direction and the wave length of the internal wave. The experiment results on SAR images shows that the proposed method can estimate the internal wave correctly.
\end{abstract}

Keywords-Internal wave;Hilber Huang Transform(HHT)); Empirical Mode Decomposition(EMD);Intrinsic Mode Function(IMF)

\section{INTRODUCTION}

Since the United States Seasat mission, much understanding has been gained form the synthetic aperture radar. It is well known that Synthetic Aperture Radar (SAR) is a powerful instrument to measure sea surface roughness, which is modulated by surface current variations. The surface current variations can be related to oceanic phenomena such as internal waves or underwater bottom topography. Thus SAR images were often used to improve our understanding of internal wave generation and evolution because of their large horizontal two-dimensional observations $^{[1]}$.

Internal waves usually have some bright and dark line or bands on the SAR images. There are many methods proposed to detect these features, Rodenas and Garello use wavelet to detect and locate the internal waves on the SAR images [2]. Marghany use digital elevation model for the detection of internal waves ${ }^{[3]}$. Warrick and Delaney use localized Radon transform to detect internal waves and some other linear features ${ }^{[4]}$.

As to parameter estimation of internal wave, there are many theories proposed to study it. Rodenas and Garello use the SAR image profiles of internal wave to estimat the half internal wave amplitude ${ }^{[5]}$. Jingsong Yang and Weigen Huang use the SAR datas to gain the depth, the wave velocity wave amplitude ${ }^{[6]}$.

Most of the detection methods need to get the dark or bright quasilinear periodic lines, but the above methods usually can't work well because the SAR images are always contaminated by significant speckles, the lines may be very thin and can be divided into several components, some of which may or may not always be visible in a SAR image.

As most of the internal waves take many pitch like curves, in this paper a novel internal wave detection and parameter estimate method based on pitch arc fitting is proposed. It just needs three points draw manually along one of the internal wave arc lines, then the parameters like the internal wave direction, the wave length and the wave amplitude can be calculated.

\section{THEORETICAL BASE}

\section{A. The characteristic of internal wave}

Thanks for the technique of SAR, the study and observation of internal wave has come up to a new step. In 1984, the ocean scientist in America take a SAR internal wave signal experiment in New York Bright, use the theory of Gasparovic, it explains the connection between the internal wave filed and the shape of internal wave in SAR.

The characteristic of internal wave and its image in SAR can be concluding as follows:

1. The internal waves are always appear as groups.

2. The internal wave front and hollow are always parallel with the ocean floor and looks like take a radiation from a point.

3. The typical distance between the bright and the dark bands are from hundreds to thousands meters, and the distance become short in the center of the radiation region.

4. The front of the internal wave may take hundreds kilometers, and it become short in the center of the radiation region.

5. The internal waves are always take bright bands in rough sea back or take dark bands in velvet sea back, which means that the internal wave can be observed in SAR image in a very wide wind regime.

\section{B. Parameter estimation}

In SAR images, the relative variation of gray scale generated by the internal waves can be described as follows, 


$$
\begin{aligned}
& \frac{\Delta I}{I_{0}}=\frac{\Delta \sigma}{\sigma_{0}}=B \operatorname{sech}^{2}\left(\frac{x^{\prime}}{l}\right) \tanh \left(\frac{x^{\prime}}{l}\right) \\
& \text { Where, } B= \pm \frac{4+\gamma}{\mu} \frac{2 C_{0} \eta_{0}}{h_{1} L} \cos ^{2} \phi
\end{aligned}
$$

From formula (1) we can see that the bright point and the dark point in a single internal wave can be calculated by formula (2).

$$
\frac{\partial}{\partial_{x}}\left(\frac{\Delta I}{I_{0}}\right)=-\frac{B}{l} \operatorname{sech}^{2}\left(\frac{x^{\prime}}{l}\right) \cdot\left[3 \tanh ^{2}\left(\frac{x^{\prime}}{l}\right)-1\right]=0
$$

The distance $x^{\prime}$ then can be derivate as $x^{\prime}= \pm 0.661 l$.The distance $D$ between the bright point and the dark point in a single internal wave can be calculated as follows.

$$
D=0.661 l
$$

When the distance $D$ has been obtained, the wave length of the internal wave can be calculate as follows.

$$
l=1.52 \mathrm{D}
$$

The wave direction is always against to the bright and gray grain, so when the bright or the gray arc of the internal wave is obtained, the wave direction can be calculated.

\section{Hilber Huang Transform}

The Hilbert Huang transform is an empirically based data-analysis method. It contain two main method, the empirical mode decomposition and the Hilbert transform.

As discussed by Huang et al., the empirical mode decomposition method is necessary to deal with data from nonstationary and nonlinear processes. The decomposition is based on the assumption that any data consists of different simple intrinsic modes of oscillations. Each intrinsic mode, linear or nonlinear, represents a simple oscillation, which will have the same number of extrema and zero-crossings. Each of these oscillatory modes is represented by an intrinsic mode function(IMF) with the following definition ${ }^{[7]}$ :

(1) In the whole dataset, the number of extrema and the number of zero-crossingsmust either equal or di?er at most by one.

(2) At any point,the mean value of the envelope defined by the local maxima andthe envelope defined by the local minima is zero.

\section{PITCH ARC FITTING ARITHMETIC}

\section{A. fitting steps}

As the internal wave are always take bright or dark arcs in the sea back, if the orient of the internal wave or the center of the circle of the arc can be found, the arc of internal wave then can be fitted.

We then summarize our method of arc fitting in the following algorithm:
Step1: Draw three points in the internal wave images along with the bright or the dark front of the internal wave.

Step2. According to the three points, calculate the center of the circle of the arc.

Step3. Calculate the radius of the arc, and draw the arc along the three points.

As long as the center and the arc is fixed, a set of crosssection data along the radius can be obtained, which may supply for the following calculation of the internal wave parameter.

\section{B. Experimental investigations}

The image data we use in the experiment is gained in 2009, which were obtained from ERS2/SAR ( C band, VV polarized, incidence angle $\approx 23^{\circ}$ ). The longitude scale is from $111^{\circ} 52^{\prime} 40 "$ to $112^{\circ} 51^{\prime} 34^{\prime \prime}$ ands the latitude scale is from $19^{\circ} 12^{\prime} 13^{\prime \prime}$ to $20^{\circ} 15^{\prime} 22 "$. The subimage which contain the internal wave feature is showed in Figure1.

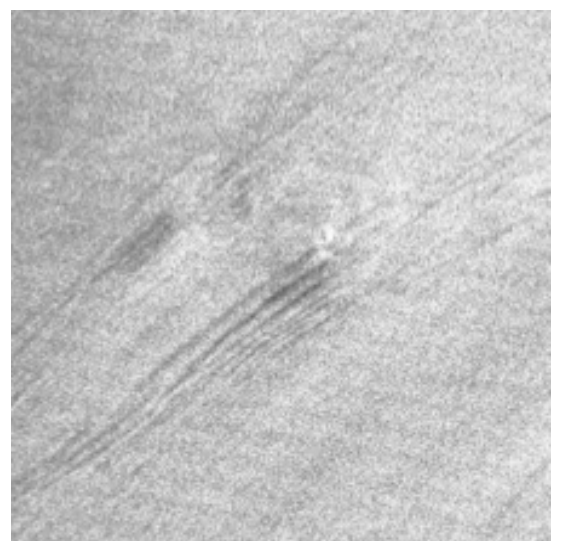

Figure 1. The Internal wave in SAR image

Following the steps we stated in III.A. we draw three points along the front of the internal wave and the arithmetic automatically extract the oriental of the internal wave and five cross sections along the direction of the internal wave, which are showed in Figure 2. Then, we automatically obtained the cross sections of internal wave showed in Figure 3.

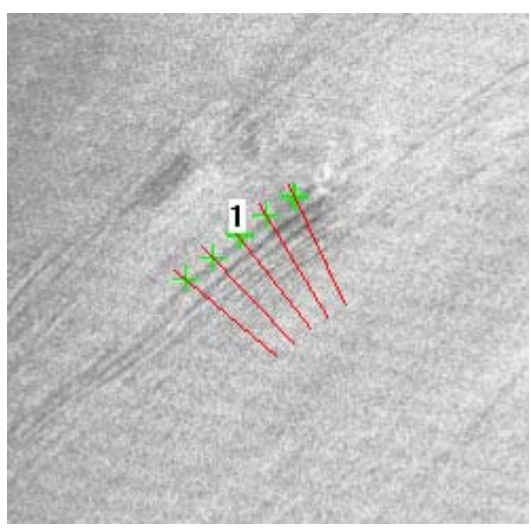

Figure 2. The curve fit result and the location of the cross sections 

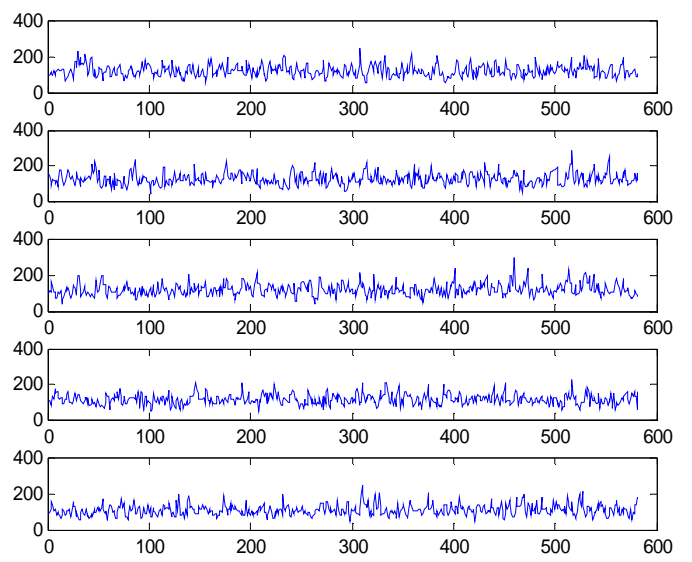

Figure 3. The cross sections of the internal wave

\section{PARAMETER ESTIMATION}

\section{A. estimation logic}

As well as the origin of the internal wave is calculated and the arc along the internal wave is located, the direction of the internal wave can be easily obtained.

The direction can be calculate by the following formula.

$$
\theta=\frac{1}{2}\left(\theta_{p 1}+\theta_{p 5}\right)
$$

Where $\theta_{p 1}$ is the angle along the internal wave center and the first point, and $\theta_{p 2}$ is the angle along the internal wave center and the fifth point.

The other parameter to estimate is the half internal wave amplitude of the internal wave. In this paper, we use the Empirical Mode Decomposition(EMD) to treat the cross sections of the internal wave.

The arithmetic is as follows.

Step1: Treat every cross section with the EMD method.

Step2. Calculate the HHT spectrum.

Step3. Extract the distance between the two typical bright bands using the HHT spectrum .

Step4. Calculate the half internal wave amplitude of the internal wave in the five cross sections use the second HHT spectrum with formula (5).

Step5. Calculate the mean half internal wave amplitude of the internal wave use the five half internal wave amplitude result calculate in step4.

\section{B. Experimental investigations}

In this paper, we use the methods descried in IV.A to calculate the wave direction and the half internal wave amplitude of the internal wave we showed in Figure.1.
The wave direction is $314.5^{\circ}$ clockwise from the north.

The HHT spectrums of the five cross sections are then calculated. Figure.4. shows the spectrums of the first cross section.

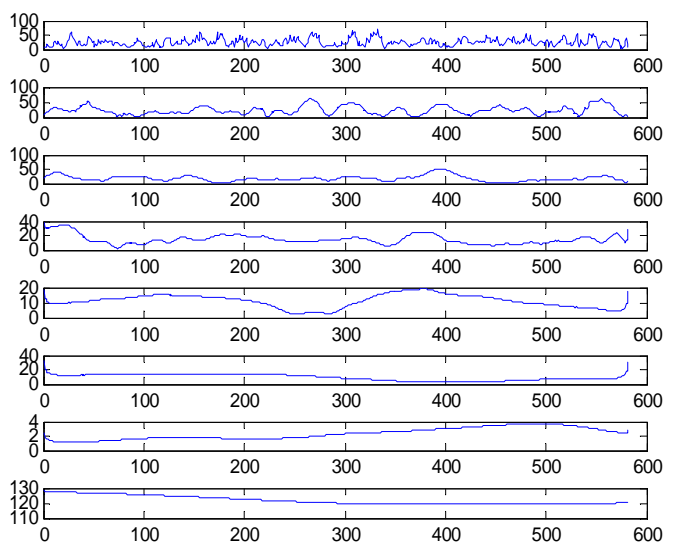

Figure 4. The spectrums of the first cross section

We can see that in the second spectrum the distance is best close to the real distribute of the bright and dark bands of internal wave in the first cross section.

The distance extract between the bright band and the dark band in the five cross sections are $0.6158 \quad 0.6551 \quad 0.4979$ 0.86470 .5503 kilometers.

Then the half internal wave amplitude calculate from the $\begin{array}{lllll}\text { five sections are } 0.9360 & 0.9958 & 0.7568 & 1.3144 & 0.8364\end{array}$ kilometers, and the final result of the internal half internal wave amplitude is 967.88 meters.

\section{CONCLUSIONS}

In this paper, a novel method to extract the internal wave parameters from SAR images is proposed, the experimental results shows that this method works well in the detection of internal waves in SAR images. Further more, using this method we can calculate the internal wave parameters easily.

The results calculated by the arithmetic in this paper can make forward using in the analyzing of the sea density and temperature level.

\section{REFERENCES}

[1] Duk-Jin Kim, SungHyun Nam, Moon, W.M., Kuh Kim. Observations of near-inertial internal waves in the East (Japan) Sea by Synthetic Aperture Radar. Geoscience and Remote Sensing Symposium, 2004. IGARSS '04. Proceedings. 2004 IEEE International Volume 3, 2004 Page(s):1896 - 1898.

[2] D.Lyden,R.R.Hammond,P.R.Lyzenga, and .A.Shuchman,”Syn thetic aperture radar imaging of surface ship wakes," J.Geophys.Res.,vol.93, no.C10,pp.12293-12303,1998.

[3] M.T.Rey,J.K.E.Tunaley,J.T.Folinsbee,,P.A.Jahans,J. A.Dixon,and M.R.Vant,"Application of Radon transform techniques to wake detection in Seasat-A SAR images” IEEE Trans.Geosct,Remote Sensing.vol.28, pp. 553-560, July,1990. 
[4] M.Atiquzzaman,M.W.Akhtar,”A tobust Hough transform technique for complete line segment dexcription”,Real Time Imaging Vol.1,No,6,pp.419-426,Dec,1995

[5] Rodenas, J.A.,Garello, R.. Wavelet analysis in SAR ocean image profiles for internal wave detection and half internal wave amplitude estimation.Geoscience and Remote Sensing, IEEE Transactions on Volume 35, Issue 4, July 1997 Page(s):933 - 945.
[6] Jingsong Yang, Weigen Huang, Chenghu Zhou, Changbao Zhou, Remote Sensing for Land \& Resources : 2003,No.1, 29-32.

[7] Norden E.Huang,Goddard Institute for Data Analter,Greenbelt,MD 20771,USA 